\title{
Erfahrungsaustausch zur neuen Screening-Methode im Bereich Wirtschaftlichkeitsprüfung
}

\author{
Patrick Müller ${ }^{\mathrm{a}}$, Thomas Kessler \\ ${ }^{a}$ Abteilungsleiter Ambulante Versorgung und Tarife FMH; ${ }^{b}$ Fachspezialist Ambulante Versorgung und Tarife FMH
}

Mitte 2018 trat der neue Vertrag betreffend Screening-Methode im Rahmen der Wirtschaftlichkeitskontrolle in Kraft. Anwendung findet diese neue Methode ab Statistikjahr 2017. Anlässlich zweier Informationsveranstaltungen haben Expertinnen und Experten die neue statistische Methode kritisch beleuchtet, und es fand ein erster Erfahrungsaustausch unter ärztlichen Interessenvertreterinnen und -vertretern statt.

Mitte 2018 trat der neue Vertrag betreffend ScreeningMethode im Rahmen der Kontrolle der Wirtschaftlichkeit gemäss Art. 56 Abs. 6 KVG zwischen FMH, curafutura und santésuisse in Kraft. Die Vertragspartner legen in diesem Vertrag als Screening-Methode zur Detektion von Ärztinnen und Ärzten mit auffälligen Kosten gemäss KVG im Sinne der verwendeten Methode die zweistufige Regressionsanalyse gemäss Schlussbericht der Studie der Polynomics AG fest. Anwendung findet diese neue Methode seit Statistikjahr 2017. Die

Es bedarf immer einer dem Screening nachgelagerten Einzelfallprüfung, ob Leistungen wirtschaftlich oder unwirtschaftlich waren.

Frage, ob ein Arzt seine Leistungen unwirtschaftlich erbringt, kann aber ausschliesslich im Rahmen einer dem Screening nachgelagerten Einzelfallanalyse unter Berücksichtigung der Praxisbesonderheiten beantwortet werden. Die Screening-Methode ist immer nur ein Teil der Wirtschaftlichkeitskontrolle gemäss Art. 56 Abs. 6 KVG und stellt keinen Ersatz für eine detaillierte Einzelfallbeurteilung dar.

Am 5.11.2020 und am 12.11.2020 fanden zwei virtuelle Informationsveranstaltungen zur neuen ScreeningMethode (Regressionsanalyse) im Bereich der Wirtschaftlichkeitsprüfung für ärztliche Vertreter von Ärzteorganisationen und Trust Center statt. Ziel dieser Informationsveranstaltungen war es, den ärztlichen Vertretern, welche betroffene Ärztinnen und Ärzte un- terstützen, begleiten und beraten, einen Überblick über diese neue Methode zu geben, wichtige Hinweise und Abläufe aufzuzeigen, ein gemeinsames Verständnis dazu zu entwickeln sowie einen Informationsaustausch unter den Anwesenden zu ermöglichen.

An den beiden Informationsveranstaltungen haben eine ausgewiesene Expertin und drei ausgewiesene Experten Referate gehalten. Dr. Andreas Kohler, Projektleiter Wirtschaftlichkeitsprüfungen tarifsuisse AG, stellte die neue Screening-Methode vor. Prof. Dr. Beat Hulliger, Dozent der FHNW im Institute for Competitiveness and Communication (ICC), gab einen Überblick über die Leistung der neuen Screening-Methode. Patrizia Gratwohl, Rechtsanwältin, gab eine juristische Einordnung und schliesslich hat Dr. Juerg B. Reust, Mandatsnehmer der FMH, die anwesenden Personen mit Informationen und Erfahrungen aus der Praxis bedient und fasste das Wichtigste zur neuen ScreeningMethode zusammen. Im Folgenden werden die Referate inhaltlich kurz aufgezeigt bzw. zusammengefasst.

\section{Grundlagen der Wirtschaftlichkeits- prüfung}

Dr. A. Kohler stellte in seinem Referat die neue Screening-Methode im Detail vor. Initial zeigte er die gesetzlichen und vertraglichen Grundlagen der Wirtschaftlichkeitsprüfung auf, wie auch die Entwicklung vom Durchschnittskostenvergleich, von ANOVA (berücksichtigt die Variablen Alter und Geschlecht) bis hin 
zum Regressionsindex (berücksichtigt zusätzlich die Variablen Spitalaufenthalt im Vorjahr, Wahlfranchise, Pharmaceutical Cost Group [PCG]). «Die Wirtschaftlichkeitsprüfung gliedert sich aus Sicht von santésuisse in drei Phasen", so Kohler, "erstens das statistische Screening, zweitens die Einzelfallprüfung und drittens die daraus resultierenden Massnahmen.» Die Grundlagen der Wirtschaftlichkeitsprüfung seien die Daten des Daten- und Tarifpools sowie die ZSR-Nummern der SASIS AG und die PCG-Liste des Bundesamtes für Gesundheit BAG. Für das Screening werde neu ein Regressionsmodell bzw. ein zweistufiges Modell mit Fixed-Effekt (Praxisstil Arzt) angewendet. "Auf der ersten Stufe wird die Morbidität der Patienten und auf der zweiten Stufe der Praxisstandort und die Facharztgruppe des Arztes berücksichtigt. Schlussendlich werden die Kosten pro Patientin oder Patient eines Arztes mit denen seiner Kollegen in derselben Facharztgruppe innerhalb der

santésuisse-Mitarbeiter versuchen teilweise, den Anschein zu erwecken, die Screening-Methode sei perfekt korrigiert und Erklärungen nicht notwendig.

gesamten Schweiz verglichen.» Das Screening sei als erster Schritt in der santésuisse-Wirtschaftlichkeitsprüfung zu verstehen und ist ein partnerschaftliches Projekt der FMH, santésuisse und curafutura. «Die Screening-Methode ist zwar wissenschaftlich fundiert, jedoch nicht perfekt und muss auch methodisch weiterentwickelt werden. Es bedarf immer dem Screening nachgelagert einer Einzelfallprüfung, um festzustellen, ob ein Arzt seine Leistungen wirtschaftlich oder unwirtschaftlich erbringt», fasst Kohler zusammen.

\section{Statistische Leistung der neuen Screening-Methode}

Prof. Dr. B. Hulliger referierte zu den Leistungen der neuen Screening-Methode. $\mathrm{Zu}$ der verwendeten Regressionsmethode merkte Prof. Hulliger an, dass «die Tests und die Vertrauensintervalle auf der Korrektheit des Modells basieren. Sofern eine wesentliche Variable vergessen wurde (Omitted Variable Bias), dann sind die Koeffizienten oft verfälscht». Die Polynomics AG habe verschiedene Variablen abgeklärt und dabei keine wesentlichen Beiträge von anderen greifbaren Variablen gefunden. "Die neue Regressionsmethode ist sicherlich gut, stellt aber keine Garantie dar, dass kein Omitted Variable Bias auftreten kann", erklärte Hulliger. Es folgte ein Vergleich mit dem mittleren marginalen Effekt. «Für den einzelnen Arzt können die vorhergesagten Kosten berechnet werden. Was nicht beim Screening berücksichtigt wird, ist z.B. die Schadensart
(Krankheit, Unfall oder Mutterschaft). Das ScreeningModell ist hoch komplex, und die Mechanik ist schwierig zu durchschauen. Die meisten Modelle enthalten sehr viele Koeffizienten.» Die Grösse dieses Modells erschwere eine genauere Untersuchung der Effekte. Weitere Variablen seien von Polynomics AG abgeklärt worden. Das bedeute aber nicht, dass ein Omitted Variable Effect ausgeschlossen werden kann. Für einen einzelnen Arzt seien jedoch die abhängigen Variablen bekannt und könnten untersucht werden. Robustheitsfragen seien im Polynomics-AG-Schlussbericht angesprochen (Winsorisierung), jedoch grundsätzliche Diagnostiken müssten mit jeder Berechnung neu untersucht werden. «Die Screening-Methode gibt Hinweise, kann aber keine Einzelfallbeurteilung ersetzen. Angemerkt sei, dass das Screening den Output der Leistungserbringung nicht berücksichtigt (Heilungserfolg)", so Hulligers Fazit.

\section{Eine Einzelfallanalyse ist weiterhin notwendig}

Rechtsanwältin Patrizia Gratwohl referierte über die rechtliche Einordnung der neuen Screening-Methode. Bei der Durchschnittskostenmethode gelte ein Indexwert von über 130 Indexpunkten als Verdacht auf Überarztung. Die Durchschnittskostenmethode sei intransparent, nicht sachgerecht bzw. kein eigentliches Wirtschaftlichkeitsverfahren, sondern lediglich ein Durchschnittskostenvergleich, welcher die Morbidität des Patientenkollektivs nicht berücksichtige, sagte Gratwohl. Diese Kritik habe zum Art. 56 Abs. 6 KVG geführt, welcher festhalte, dass die Leistungserbringer und Versicherer vertraglich eine Methode zur Kontrolle der Wirtschaftlichkeit festzulegen hätten. «Die Zielsetzung von Art. 56 Abs. 6 KVG ist, Transparenz bezüglich der Kontrollmethode zu schaffen, die Morbidität des Patientenkollektivs zu berücksichtigen sowie eine Methode zur Kontrolle der Wirtschaftlichkeit, und nicht zur Umsetzung der Wirtschaftlichkeitsprüfung als solche, vertraglich zu regeln", so Gratwohl. Im Vertrag nach Art. 56 Abs. 6 KVG zwischen curafutura, FMH und santésuisse sei festgehalten, dass mit der Screening-Methode zur Detektion von Ärzten mit auffälligen Kosten gemäss KVG noch nicht bewiesen sei, ob der Arzt unwirtschaftlich arbeitet oder nicht. «Dies kann erst in der nachgelagerten Einzelfallanalyse erfolgen.» Zur Rechtsprechung äusserte Gratwohl zudem, dass bislang noch keine höchstrichterliche Rechtsprechung zur Screening-Methode erfolgt sei und dass die Frage nach der rechtlichen Einordnung der Screening-Methode noch offen sei. Die Screening-Methode diene als Methode zur Identifikation von Ärzten mit 
statistisch auffälligen Kostenprofilen, und die Wirtschaftlichkeitsprüfung sei im Einzelfall unter Berücksichtigung von Praxisbesonderheiten vorzunehmen im Sinne einer Kombination der statistischen und analytischen Methode.

\section{Vorbeugen ist besser als kurzfristiges Reagieren}

Dr. J. B. Reust ging in seinem Referat hauptsächlich auf wichtige Erkenntnisse ein, die aufgrund der nun zweieinhalbjährigen Erfahrung mit der neuen ScreeningMethode gemacht wurden. «Die Hauptziele wie die Reduktion der auffälligen Ärzte und bessere Berücksichtigung der Morbidität wird mit der neuen Screening-Methode erreicht», bestätigte Reust. Es gelte aber insbesondere zu beachten, dass die Screening-Methode nicht für alle Praxisbesonderheiten korrigiert und nur ein erster Schritt zur Detektion von Ärzten mit auffälligen Kosten sei. Ein Arzt tue gut daran, sich vorzubereiten, d.h. den Regressionsbericht mit Kostendaten und Indizes bei santésuisse zu bestellen und eine allfällig notwendige Argumentation vorzubereiten. Es gebe keine Reaktionszeit mehr, d.h., in einzelnen Fällen habe santésuisse den Arzt schon im ersten Jahr eingeklagt.

Zur Argumentation stünden neben dem Regressionsbericht die eigenen Praxisdaten zur Verfügung. Diese sollten nach verschiedenen Kriterien (z.B. Bildung von Kostengruppen je nach Indikation) analysierbar sein. Ebenfalls eignete sich der Praxisspiegel gut zur Unterstützung der Argumentation. Deshalb: «Abrechnungsdaten in die ärzteeigene Datensammlung (via Trust Center oder intermediär wie Ärztekasse, MediData) liefern. Neben Alter und Geschlecht ist insbesondere der Einfluss der PCG auf den Regressionsindex sehr gross", erklärte Reust. Die neuen Morbiditätskriterien «Anteil hohe Franchise» und "Spitalaufenthalt im Vorjahr» würden im Allgemeinen einen geringeren Einfluss haben, so Reust weiter. Mit den zur Verfügung stehenden Daten sei nicht ersichtlich, welcher Morbiditätsparameter dominant ist. Immerhin liessen sich durch den Vergleich des unkorrigierten Indexes, des ANOVA-Indexes und des Regressionsindexes "Totale Kosten» die Auswirkungen qualitativ beurteilen.

Eine weitere Schwierigkeit bestehe darin, dass die relevanten PCG-Gruppen und die darin relevanten Medikamente nicht konstant seien, sondern jährlich wechseln würden. Es könne also sein, dass bei einem Arzt bei identischer medikamentöser Behandlung in einem Jahr der Regressionsindex zugunsten des Arztes korri- giert wird, im Folgejahr jedoch zu dessen Ungunsten. Bei der Einführung einer neuen Methode gebe es immer auch Verlierer. Eine Gruppe seien z.B. komplementär tätige Fachärzte für Allgemeine und Innere Medizin, die generell wenig Medikamente abgeben oder verordnen. In diesen Fällen würde der Regressionsindex Totale Kosten nach oben, d.h. zu Ungunsten des Arztes korrigiert. «Die Systemannahme ist: wenig Medikamente gleich nicht morbides Patientengut mit tiefen Kosten (pro memoria: vereinfachte Aussage). Und alle, auch santésuisse, machen Fehler. Deshalb ist es ratsam, den Regressionsbericht von santésuisse mit den eigenen Praxisdaten und dem Praxisspiegel zu plausibilisieren", empfahl Reust. Zum Beispiel wenn die Daten des Behandlungsdatums gegenüber dem Abrechnungsdatum beim Versicherer nicht identisch seien, so Reust. Diese sollten aber natürlich in etwa übereinstimmen. Mit einer gut vorbereiteten Argumentation liessen sich Praxisbesonderheiten respektive das Kostenbild erklären - dies benötige aber entsprechend Zeit. Die santésuisse-Mitarbeiter versuchten zum Teil den Anschein zu erwecken, dass die Screening-Methode perfekt korrigiert sei und gar keine Erklärungen mehr notwendig seien. «Dies ist nicht nur entgegen dem Vertragswortlaut, sondern zeigt Situationen auf, in denen die Screening-Methode schlicht falsch korrigiert sind», so das Fazit von Reust.

\section{Meldung von interessanten Fällen und Erkenntnissen}

Die Screening-Methode muss auch künftig weiterentwickelt und weiter verfeinert werden. Dafür setzt sich die FMH in den Verhandlungen mit santésuisse und curafutura ein. Der Austausch unter den beteiligten Interessenvertretern im Bereich der Wirtschaftlichkeitsprüfung wird seitens FMH auch künftig gefördert werden. Bitte melden Sie uns interessante und besondere Fälle, Auffälligkeiten und Verhalten seitens tarifsuisse AG, bzw. melden Sie uns weiter wichtige Erkenntnisse, Erfahrungen und mögliche Ansatzpunkte. Zudem können Sie gerne mit konkreten Vorschlägen, Fragen und Anliegen auf die FMH zukommen (tarife.ambulant[at] fmh.ch). Reale Fälle aus der Praxis und Probleme der Methode zu erkennen heisst, Ansatzpunkte für die weitere Verfeinerung und Weiterentwicklung der Methode ableiten zu können. Die FMH empfiehlt betroffenen Ärztinnen und Ärzten eines Wirtschaftlichkeitsverfahrens, sich mit ihrer kantonalen Ärztegesellschaft oder ihrem Trust Center in Verbindung zu setzen. 